\title{
OIL-INFUSED SUPERHYDROPHOBIC SILICON SURFACES FOR ENHANCED WATER CONDENSATION HEAT TRANSFER
}

\author{
R. Xiao ${ }^{l}$, R. Enright ${ }^{1,2}$, N. Miljkovic ${ }^{l}$, and E. N. Wang $^{l^{*}}$ \\ ${ }^{1}$ Department of Mechanical Engineering, MIT, Cambridge, Massachusetts, USA \\ ${ }^{2}$ Stokes Institute, University of Limerick, Limerick, Ireland
}

\begin{abstract}
Superhydrophobic surfaces have received significant interest for dropwise condensation to increase the efficiency of energy applications such as heat exchangers, power plants, and solar thermal energy conversion systems. While superhydrophobic surfaces can be fabricated using silane self-assembled coatings (SAC) on oxide structures, significant heat transfer enhancements require high nucleation densities that are difficult to achieve due to the high energy barrier for nuclei formation. In this work, we demonstrated that by infusing microstructured surfaces with lowsurface tension oil, nucleation densities can be increased by over an order of magnitude while maintaining low droplet adhesion. This work offers a simple and scalable approach to create surfaces that can be tailored for enhanced heat transfer.
\end{abstract}

\section{INTRODUCTION}

Condensation heat transfer has wide applications in various systems such as heat exchangers, heat pipes and power plants. The heat transfer coefficient of condensation is of great significance to the efficiency of such systems. Dropwise condensation, where the condensate forms discrete droplets rather than continuous films covering the substrate, is considered as one of the most promising approaches to enhance the heat transfer coefficient. Previous work has demonstrated the application of nanostructured superhydrophobic surfaces where condensate can be spontaneously removed via a surface-tension-driven mechanism [1]. However, the nucleation density on these surfaces is relatively low since the phase change process relies on high energy active sites to initiate nucleation at low supersaturations (low $\Delta T$ ), limiting the overall heat transfer performance. Furthermore, air pockets trapped beneath the droplets during growth reduce the contact area between the condensing droplet and substrate, which increases the thermal resistance and reduces the heat transfer coefficient [2].

Recently, Wong et al. [3] demonstrated a liquid-solid composite surface created by infusing a porous fluoropolymer with water-immiscible, low-surface-tension Krytox oil. On such a composite surface, the contact area between droplet and substrate can be large while contact line pinning remains very low allowing easy removal of droplets. These properties make the surfaces potentially suitable for enhanced condensation heat transfer. While such behavior is possible with the proper choice of silane, e.g., dichlorodimethylsilane on $\mathrm{SiO}_{2}$, the nucleation density is limited by the presence of high surface energy defects and contaminants at low supersaturations (low $\Delta T$ ). In this work, we show that the nucleation density on oil-infused, silane-coated structured surfaces can be significantly increased by the use of disordered long-chain silane coatings that result in nucleation sites limited only by the density of pillar structures comprising the surface. We find that the increase in the nucleation density can be explained by heterogeneity in the surface energy of the silane coating and the reduced water-oil interfacial energy. This effect could potentially be used to significantly improve the heat transfer coefficient in condensation by controlling the nucleation density.

\section{FABRICATION}

Well-defined silicon micro/nanopillar arrays with diameters, $d$, ranging from $0.4 \mu \mathrm{m}$ to $5 \mu \mathrm{m}$, periods, $l$, ranging from $4 \mu \mathrm{m}$ to $25 \mu \mathrm{m}$, and heights, $h$ ranging from $10 \mu \mathrm{m}$ to $25 \mu \mathrm{m}$ were used in our experiments. The silicon surfaces were functionalized with three different chemicals: 1) (Tridecafluoro-1,1,2,2tetrahydrooctyl)-1-trichlorosilane (TFTS) [UCT Specialties], which forms a self-assembled coating (SAC) by chemical vapor deposition (CVD) with a relatively long carbon chain $(\mathrm{MW}=$ $481.54 \mathrm{~g} / \mathrm{mol}$ ), 2) Dimethyldicholorosilane (DMCS) [SigmaAldrich], which forms a self-assembled monolayer (SAM) by CVD with a short carbon chain $(\mathrm{MW}=129.06 \mathrm{~g} / \mathrm{mol})$, and 3) Poly $(1 \mathrm{H}, 1 \mathrm{H}, 2 \mathrm{H}, 2 \mathrm{H}-$ perfluorodecyl acrylate) (PFDA) polymer, which was deposited using initiated chemical vapor deposition (iCVD) with a typical film thickness of $35 \mathrm{~nm}$. Goniometric measurements on smooth functionalized silicon surfaces showed advancing and receding contact angles of: $\theta_{\mathrm{a}} / \theta_{\mathrm{r}}=122^{\circ} \pm 1.3^{\circ} / 78^{\circ}$ $\pm 1.3^{\circ}$ (equilibrium contact angle $\theta_{\mathrm{e}} \approx 102.1^{\circ} \pm 0.9^{\circ}$ ); $\theta_{\mathrm{a}} / \theta_{\mathrm{r}}=$ $103.8^{\circ} \pm 0.5^{\circ} / 102.7^{\circ} \pm 0.4^{\circ}\left(\theta_{\mathrm{e}} \approx 103.2^{\circ} \pm 0.3^{\circ}\right) ;$ and $\theta_{\mathrm{a}} / \theta_{\mathrm{r}}=$ $121.1^{\circ} \pm 2.2^{\circ} / 106.3^{\circ} \pm 2.4^{\circ}\left(\theta_{\mathrm{e}} \approx 113.5^{\circ} \pm 1.6^{\circ}\right)$ for deposited films of TFTS, DMCS, and PFDA respectively. A small droplet of Krytox GPL 100 oil [DuPont] was applied to the functionalized silicon pillar arrays. The surface tension of Krytox oil is $\sim 17-$ $19 \mathrm{mN} / \mathrm{m}$, allowing the oil to spread on the surface. A dry nitrogen stream was used to assist spreading and remove excess oil. Typical scanning electron micrographs (SEM) of the silicon pillar arrays without and with the oil are as shown in Fig. 1(a) and (b).

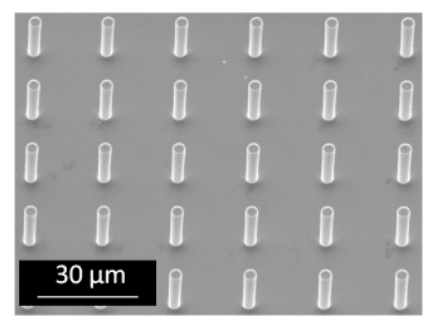

(a)

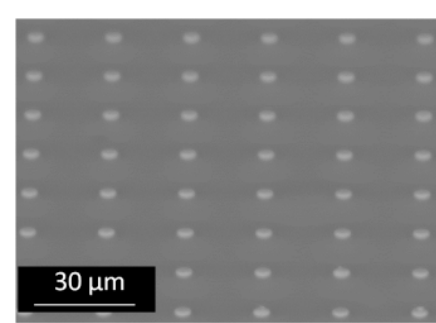

(b)
Fig. 1 Scanning Electron Micrographs (SEM) of a pillarstructured silicon surface $(d=5 \mu m, l=25 \mu m, h=15 \mu m)$ (a) without and (b) with the oil infusion.

We investigated the nucleation behavior on the surfaces with and without the oil under white light optical microscopy (OM). The samples were horizontally mounted on a thermal stage [Instec Inc.] inside an enclosure and cooled to $T_{\mathrm{w}}=283.1 \pm 0.1 \mathrm{~K}$ in a dry nitrogen atmosphere. Following thermal equilibration $(\sim 5 \mathrm{~min})$, nucleation was initiated by flowing water-saturated nitrogen into the enclosure. The supersaturation, defined as the ratio of the vapor pressure to the saturation pressure at the stage temperature $\left(p_{\mathrm{v}} / p_{\mathrm{w}}\right)$, was controlled by the temperature of the water reservoir through which the nitrogen carrier gas was sparged and measured using a humidity probe [Hygroclip, Rotronic] located $\sim 1 \mathrm{~cm}$ above the sample. Typical values of the supersaturation were around $S \approx 1.6$. The nucleation density and subsequent growth behavior was recorded at a frame rate of $30 \mathrm{fps}$ using a CMOS camera [Phantom V7.1, Vision Research] attached to the optical microscope. As 
shown in Fig. 2(a) and (b), the nucleation density on oil-infused surface with long-chain TFTS coating was significantly higher than that on the same surface without oil. We did not observe any nucleation between pillars which can be attributed to the large thickness of oil coverage between pillars that introduces a large diffusion resistance for water vapor. However, a droplet was formed on the tip of almost each pillar where the oil film was thin enough to allow sufficient mass to diffuse to the functionalized silicon surface. Meanwhile, on pillar arrays coated with DMCS and PFDA, we did not observe similar increases in nucleation density, as shown in Fig. 2(c).

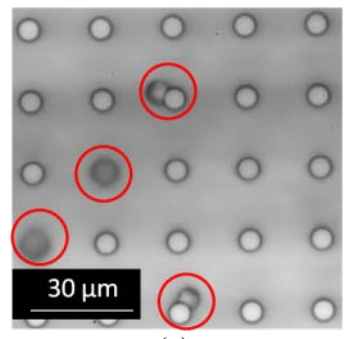

(a)

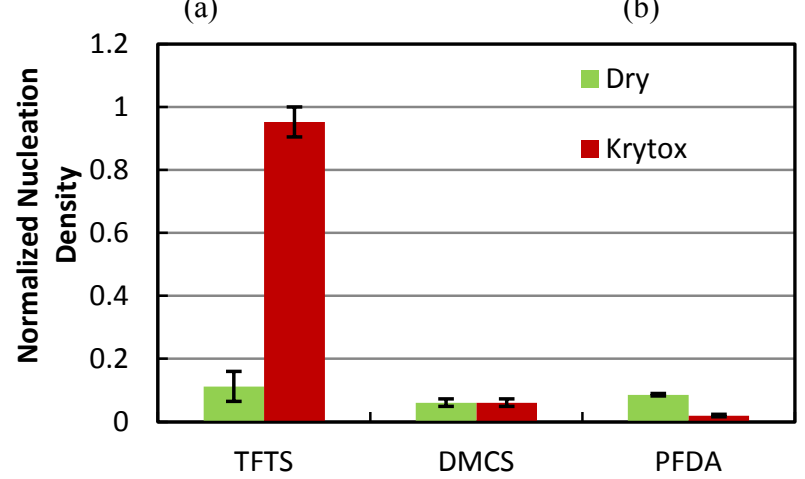

(c)

Fig. 2(a) White-light microscopy image of condensation on a silicon pillar array without oil, with nucleation sites highlighted by red circles. (b) White-light microscopy image of condensation on a composite surface with oil. Nucleation occurred on the tip of almost every pillar. (c) Comparison of the change in nucleation density with various surface coatings. The nucleation densities were normalized against pillar densities for a fair comparison.

\section{AFM IMAGING AND CONTACT ANGLE ANALYSIS}

To investigate the mechanism for this drastic change in nucleation density, we performed atomic force microscopy (AFM) in tapping mode on a smooth TFTS-coated silicon surface and observed the presence of micelle structures, as shown in Fig. 3(a). Such micelle structures have been observed in previous studies and considered as disordered agglomeration of excessive silane molecules [4]. The phase image of the AFM measurement (Fig. 3(d)) showed significantly higher phase angle on the micelle structures, which suggests that the micelles were locally more hydrophilic compared to the background film. Such heterogeneity was also supported by the high contact angle hysteresis $\left(\theta_{\mathrm{a}} / \theta_{\mathrm{r}}=\right.$ $\left.122^{\circ} \pm 1.3^{\circ} / 78^{\circ} \pm 1.3^{\circ}\right)$. However, such micelle structures were not observed on other kinds of surface coatings such as DMCS and PFDA, as shown in Fig. 3(b) and (c).

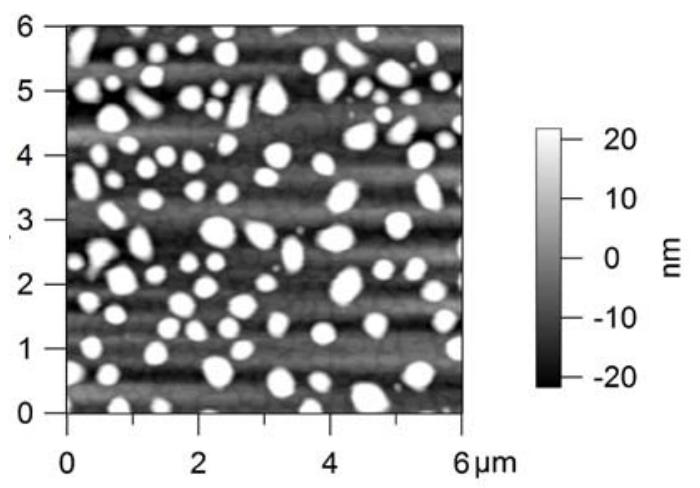

(a)

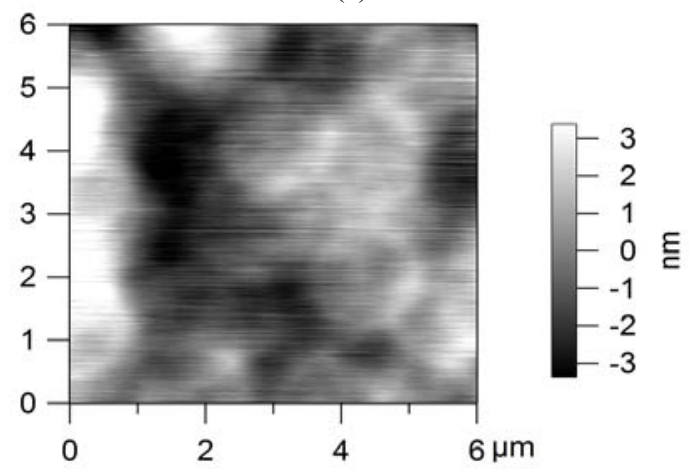

(b)

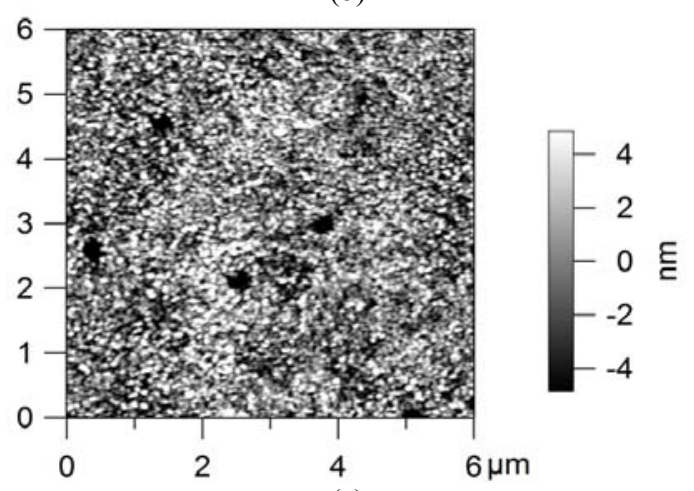

(c)

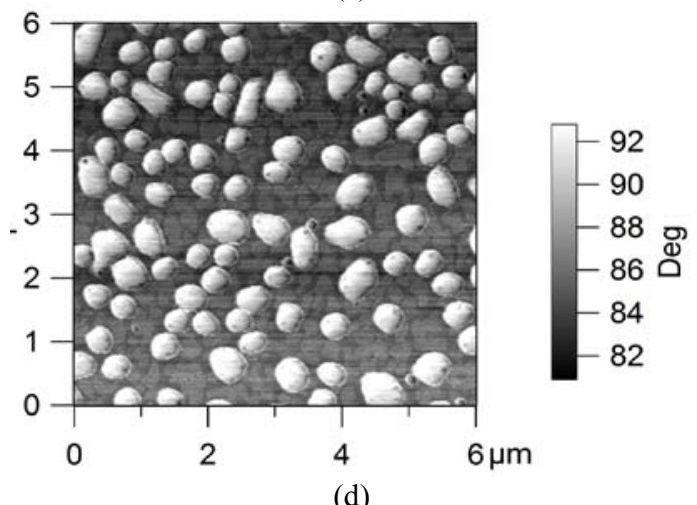

Fig. 3 Atomic Force Microscopy (AFM) height images of smooth silicon surfaces coated with (a) TFTS, (b) DMCS, and (c) PFDA. Micelles structures were only observed on TFTS coated surfaces. (b) AFM phase image of smooth silicon surface coated with TFTS. The local high phase angle indicates higher hydrophilicity of micelles. 
The local contact angles on the hydrophobic substrate and the micelle structures can be determined based on a modified CassieBaxter model. Assuming the local contact angles on the hydrophobic substrate and the micelle structures to be $\theta_{1}$ and $\theta_{2}$, respectively, the macroscopic advancing and receding contact angles are determined as

$$
\begin{gathered}
\theta_{a}=\theta_{1}, \\
\cos \theta_{r}=\sqrt{f} \cos \theta_{2}+(1-\sqrt{f}) \cos \theta_{1},
\end{gathered}
$$

where $f$ is the area fraction of the micelles [5].

Based on the macroscopically measured advancing and receding angles, $\theta_{\mathrm{a}}=122^{\circ} \pm 1.3^{\circ}$ and $\theta_{\mathrm{r}}=78^{\circ} \pm 1.3^{\circ}$, and the fraction of the micelles determined as $f \approx 0.4$ from AFM, the local contact angles on the hydrophobic substrate and the hydrophilic micelles were found to be $\theta_{1}=122^{\circ} \pm 1.3^{\circ}$ and $\theta_{2}=60^{\circ} \pm 1.5^{\circ}$.

\section{CLASSICAL NUCLEATION THEORY}

The nucleation rate, $J$, can be determined by classical nucleation theory (CNT) as [6]

$$
J=z f^{*} \exp \left(-G^{*}\right)
$$

In Eqn. (3), $z$ is the Zeldovich factor and $G^{*}$ is the dimensionless energy barrier, given by

$$
\begin{aligned}
z & =(k T \ln S)^{2} / 8 \pi v_{o} \sqrt{k T \psi(\theta) \gamma^{3}} \\
G^{*} & =16 \pi \psi(\theta) v_{o}^{2} \gamma^{3} / 3(k T)^{2}(\ln S)^{2}
\end{aligned}
$$

where $S$ is the supersaturation and $\psi(\theta)$ is the activity that accounts for the effect of contact angle. $f^{*}$ is the frequency of monomer attachment to the critical droplet nucleus dependent on the nature of the nucleus growth. The main modes of growth during heterogeneous nucleation are limited via surface diffusion or direct impingement of monomers to the nucleus [7, 8]. Volumetric diffusion is a third growth limiting step, which is only considered important for nucleation taking place in liquid or solid solutions [6]. However, we included all three mechanisms when calculating the nucleation rates.

The frequency of monomer attachment due to direct vapor impingement is given by

$$
f^{*}{ }_{i}=\gamma_{\mathrm{n}}\left[\left(1-\cos \left(\theta_{\mathrm{w}}\right)\right) / 2 \psi^{2 / 3}(\theta)\right]\left(36 \pi v_{\mathrm{o}}^{2}\right)^{1 / 3} \operatorname{In}^{2 / 3}
$$

where $\gamma_{\mathrm{n}}$ is the sticking coefficient $\left(0<\gamma_{n}<1\right), I$ is the classical Hertz-Knudsen impingement rate $\left.\left(I=P / \sqrt{2 \pi m_{0} k T}\right)\right), n$ is the number of molecules in the nucleated cluster, and $v_{\mathrm{o}}$ is the volume of an individual water molecule $\left(v_{0}=3 \times 10^{-29} \mathrm{~m}^{3}\right)$. To determine an upper bound on the nucleation rate, a sticking coefficient of one was assumed $\left(\gamma_{\mathrm{n}}=1\right)$.

The frequency of monomer attachment due to surface diffusion is given by $f^{*}{ }_{\mathrm{sd}}=\gamma_{\mathrm{n}} c^{*} \lambda_{\mathrm{s}}{ }^{2} I$, where $c^{*}$ is the capture number due to surface diffusion $\left(1<c^{*}<5\right)$, and $\lambda_{\mathrm{s}}$ is the mean surface diffusion distance of an adsorbed monomer on the substrate. The capture number $c^{*}$ is size independent and approximately equal to 1.9 for heterogeneous condensation of water vapor [9]. The mean surface diffusion distance is dependent on the wettability of the substrate and is given by $\lambda_{\mathrm{s}}=\sqrt{D_{\mathrm{sd}} \tau_{\mathrm{d}}}$ where $D_{\text {sd }}$ is the surface diffusion coefficient $\left(D_{\mathrm{sd}}=d_{\mathrm{s}}{ }^{2} v_{\mathrm{s}} \exp \left[-E_{\mathrm{sd}} / k T\right]\right), \tau_{\mathrm{d}}$ is the desorption time $\left(\tau_{\mathrm{d}}=\right.$ $\left.\left(1 / v_{\mathrm{s}}\right) \exp \left[-E_{\mathrm{des}} / k T\right]\right), v_{\mathrm{s}}$ is the adsorbed molecule vibration frequency determined using the Debye approximation $\left(v_{\mathrm{s}}=\right.$ $\left.V_{D} a / 2\right), d_{\mathrm{s}}$ is the length of a molecular jump along the substrate surface approximated by the lattice constant of the substrate $\left(d_{\mathrm{S}}=5.4 \AA\right)$ [10] and $V_{\mathrm{D}}$ is the speed of sound in the substrate $\left(V_{\mathrm{D}}=8433 \mathrm{~m} / \mathrm{s}\right)$. The desorption and surface diffusion energies are given by $E_{\mathrm{des}}=E_{1}+\sigma_{\mathrm{sv}} a_{\mathrm{o}}$ and $E_{\mathrm{sd}}=0.5 E_{\mathrm{des}}$ [11], respectively, where $E_{l}$ is the binding energy of an $n=1$ sized cluster, $\sigma_{\mathrm{sv}}$ is the solid vapour interfacial energy and $a_{\mathrm{o}}$ is the water molecule surface area $\left(a_{\mathrm{o}}=4.67 \times 10^{-19} \mathrm{~m}^{2}\right)$. The calculated energies of desorption show excellent agreement with that of experiment and molecular dynamics simulations $\left(E_{\mathrm{des}, \mathrm{SiO} 2}=0.9 \mathrm{eV}\right)[12,13]$.

The frequency of monomer attachment due to volumetric diffusion is given by

$$
f^{*}{ }_{\mathrm{vd}}=\gamma_{\mathrm{n}}\left(1-\frac{\cos \theta_{\mathrm{w}}}{\psi^{1 / 3}}\right)\left(6 \pi^{2} v_{\mathrm{o}}\right)^{1 / 3} D C n^{1 / 3}
$$

where $D$ is the self diffusion coefficient of water vapor $(D=$ $\left.\left(3 / 8 \pi n_{\mathrm{o}}{d_{\mathrm{o}}}^{2}\right) \sqrt{k T / \pi m_{\mathrm{o}}}\right), C$ is the equilibrium concentration of monomers $\left(C=\left(1 / a_{0}\right) \exp \left(-W_{1} / k T\right)\right), d_{0}, m_{0}$ and $n_{0}$ are the water molecule diameter $\left(d_{\mathrm{o}}=3.0 \AA\right)[12]$, mass $\left(m_{\mathrm{o}}=3 \times 10^{-26} \mathrm{~kg}\right)$ [6] and number density $\left(n_{\mathrm{o}}=N_{\mathrm{A}} / v_{\mathrm{M}}\right)$, respectively.

By adding the nucleation rate from the three mechanisms together, the nucleation rate, $J$, can be determined as a function of the contact angle and surface tension of the condensate at given supersaturations, as shown in Fig. 4.

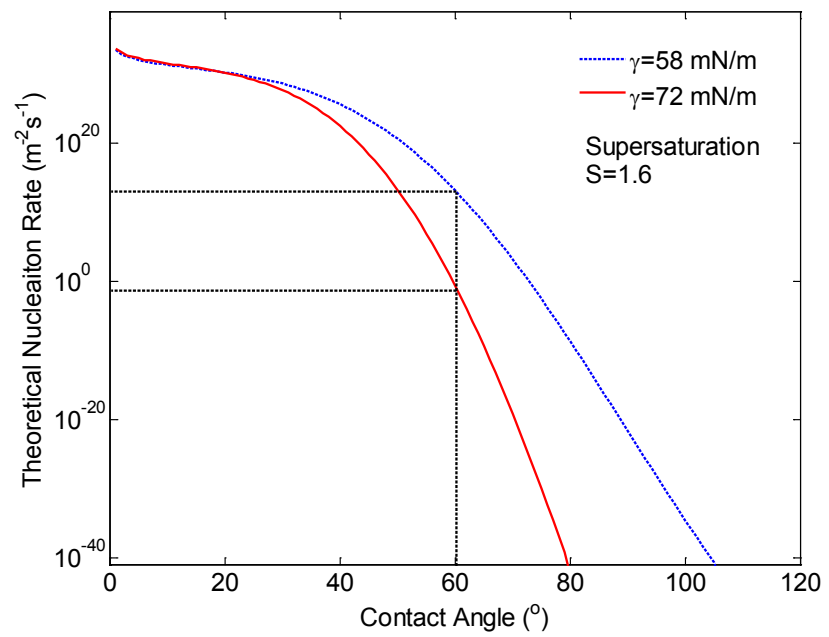

Fig. 4 Nucleation rate predicted by the classical nucleation theory as a function of contact angle and interfacial tension at $S=1.6$.

The surface tension of water in air is $72 \mathrm{mN} / \mathrm{m}$ and the interfacial tension between water and the Krytox oil was found to be $58 \mathrm{mN} / \mathrm{m}$ by measuring the contact angle of water droplet on oil film. From Fig. 4 we can see that for the hydrophilic micelles $(\theta \approx$ $60^{\circ}$ ), the reduced interfacial tension between water and oil leads to a significant increase in the nucleation rate. With the micelles acting as nucleation sites, nucleation was supposed to occur on almost every pillar tip where the oil film was thin enough for water vapor to diffuse through. On surfaces without the micelles, such as DMCS and PFDA-coated surfaces, the contact angles are over $100^{\circ}$ and the nucleation rate was essentially zero even with reduced interfacial tension. 
In order to validate our assumption, we carried out condensation experiments using silicon pillar arrays coated with 3(trimethoxysilyl)propyl methacrylate (3-TMPM). The advancing and receding contact angle of water on a smooth silicon surface coated with $3-\mathrm{TMPM}$ are $65^{\circ} \pm 1.5^{\circ}$ and $53^{\circ} \pm 1.1^{\circ}$, respectively. The contact angle is in the range where the nucleation rate will be almost zero with a surface tension of $72 \mathrm{mN} / \mathrm{m}$ and nucleation should occur on every tip of pillars with an interfacial tension of 58 $\mathrm{mN} / \mathrm{m}$. Optical images of the condensation experiments are as shown in Fig. 5. Similar to the behavior of TFTS-coated surface, a significant increase in nucleation density was observed with the addition of Krytox oil as expected. This result supports well our hypothesis related to the role of the hydrophilic micelle structures in the droplet nucleation process.

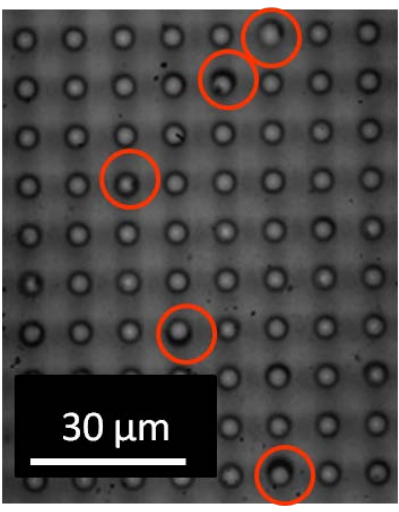

(a)

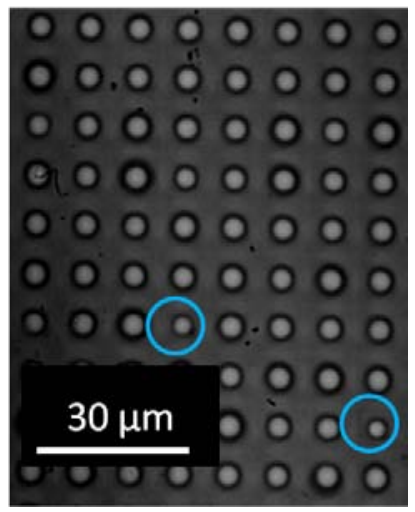

(b)
Fig. 5 Optical microscope images of the condensation experiments on 3-TMPM coated silicon pillar arrays (a) without $\left(N=6.7 \times 10^{8} \mathrm{~m}^{-2}\right)$ and (b) with Krytox oil $\left(N=1.7 \times 10^{10} \mathrm{~m}^{-2}\right)$. Nuclei are highlighted by red circles in (a) and pillars without nucleation were highlighted by blue circles in (b). The pillars have diameters of $2.5 \mu \mathrm{m}$, periods of $7.5 \mu \mathrm{m}$ and heights of $25 \mu \mathrm{m}$ $\left(l^{-2}=1.7 \times 10^{10} \mathrm{~m}^{-2}\right)$.

\section{SUMMARY}

In summary, we observed over an order of magnitude increase in the nucleation density on hydrophobic silicon pillar arrays coated with a long-chain silane molecule when hydrophobic oil was introduced on the surface. AFM imaging revealed the existence of locally hydrophilic micelles despite the overall hydrophobicity of the silane self-assembled coating (SAC). The increased nucleation density is explained in the context of classical nucleation theory as the combined effect of the hydrophilic micelles and the reduction in interfacial energy between water and oil. Control experiments on silicon pillar arrays with hydrophobic coatings without micelles and hydrophilic coatings were performed to support our findings. Such phenomena could potentially be used to create surfaces for enhanced condensation heat transfer for a variety of thermal and energy systems.

\section{ACKNOWLEDGEMENT}

The authors gratefully acknowledge the help of Rong Yang from Prof. K.K.Gleason's group at the Department of Chemical Engineering at MIT for deposition of PFDA polymer. The authors would also like to acknowledge the funding support from the Office of Naval Research (ONR) with Dr. Mark Specter as the project manager. R. Enright also acknowledges funding received from the Irish Research Council for Science, Engineering, and
Technology, co-funded by Marie Curie Actions under FP7. The authors would also like to thank the MIT Microsystems Technology Lab for fabrication staff support, help, and use of equipment.

\section{REFERENCES}

[1] J.B.Boreyko and C.-H. Chen, "Self-Propelled Dropwise Condensate on Superhydrophobic Surfaces", PRL, 2009. 103(18): p. 184501

[2] N. Miljkovic, R. Enright, and E.N. Wang, "Effect of Droplet Morphology on Growth Dynamics and Heat Transfer during Condensation on Superhydrophobic Nanostructured Surfaces", ACS Nano, 2012 6(2): p. 1776-1785

[3] T.-S.Wong, S.H.Kang, S.K.Y.Tang, E.J.Smythe, B.D.Hatton, A.Grinthal and J.Aizenberg, "Bioinspired self-repairing slippery surfaces with pressure-stable omniphobicity", Nature, 2011. 477: p. 443-447.

[4] B. C. Bunker, R. W. Carpick, R. A. Assink, M. L. Thomas, M. G. Hankins, J. A. Voigt, D. Sipola, M. P. de Boer, and G. L. Gulley, "The Impact of Solution Agglomeration on the Deposition of Self-Assembled Monolayers", Langmuir, 2000, 16 (20), pp 7742-7751

[5] R. Raj, unpublished, 2012

[6] D. Kashchiev, Nucleation: Basic Theory with Applications. 1 ed. 2000, Oxford: Butterworth-Heinemann.

[7] G.M.Pound, M.T. Simnad, and L. Yang, "Heterogeneous nucleation of crystals from vapor" J. Chem. Phys., 1954. 22(1215).

[8] R.A.Sigsbee, "Atom capture and growth rates of nuclei", JAP, 1971. 42(10): p. 3904-3915.

[9] D.J. Pocker, and S.J. Hruska, "Detailed calculations of the numberof distinct sites visited in random walk on several twodimensional substrate lattices." J. Vac. Sci. Tech., 1971. 8(6): p. 700-707.

[10] J.P.Hirth, and G.M. Pound, Condensation and evaporation nucleation and growth kinetics. 1963, England: Pergamon Press.

[11] P.A.Thiel, and T.E. Madey, "The interaction of water with solid surfaces: Fundamental aspects." Surface Science Reports, 1987. 7(6-8): p. 211-385.

[12] J.N. Israelachvili, Intermolecular and surface forces. 2nd ed. 1991, Amsterdam: Academic Press.

[13] Y.Ma, A.S. Foster, and R.M. Nieminen, "Reactions and clustering of water with silica surface". J. Chem. Phys., 2005. 122(144709)

\section{CONTACT}

*E.N. Wang, tel: +1-617-324-3311; enwang@mit.edu 